\title{
Symptomatic papillary tumour of the pulmonary valve
}

\author{
LINDA CAROL COOPER \\ M.R.C.P. (U.K.) \\ Queen Elizabeth II Hospital, Welwyn Garden City, Herts
}

\begin{abstract}
Summary
Papillary 'tumours' are small pedunculated lesions of the valve cusps and result from wear-and-tear. They are therefore frequent post-mortem findings in older patients. The vast majority are clinically silent, though occasionally such lesions when on the aortic valve may produce symptoms of myocardial or cerebral ischaemia. None has been known to cause symptoms when in the right side of the heart.

A 52-year-old woman is reported who had transient episodes of right ventricular outflow obstruction related to the presence of a papillary tumour on the pulmonary valve. Extensive radiotherapy to the chest wall was thought to be a causal factor.
\end{abstract}

\section{Introduction}

Papillary 'tumours' are not uncommon on the heart valves of older patients and are associated with wear-and-tear or with previous inflammation. Most arise in the left heart, and the vast majority are clinically silent. In this report, a 52-year-old woman presented with transient episodes of right ventricular outflow obstruction apparently caused by a pedunculated papillary tumour of the pulmonary valve. Such an association has not been described previously.

\section{Case report}

A 52-year-old woman was admitted with a 5-week history of general malaise and increasing shortness of breath. Seventeen years previously she had had a left radical mastectomy and bilateral oophorectomy. Postoperative radiotherapy had resulted in gross deformity of the left chest wall and multiple sinuses.

On admission she had a sinus tachycardia and left parasternal heave. There was a grade $3 / 4$ ejection systolic murmur heard all over the precordium. Coarse inspiratory crackles were present over the left lung field, and were attributed to radiation fibrosis. The haemoglobin was $12.5 \mathrm{~g} / \mathrm{dl}$ and the erythrocyte sedimentation rate $32 \mathrm{~mm} / \mathrm{hr}$.
In the following week she developed several episodes of shortness of breath, with hypotension $(90 / 60 \mathrm{mmHg})$, a greatly elevated jugular venous pressure, and a gallop rhythm. The electrocardiogram showed a sinus tachycardia. There was no evidence of pulmonary oedema on the chest X-ray. M-mode echocardiography showed no abnormality on the left side of the heart; the pulmonary valve was not visualized.

Each of these attacks arose spontaneously at rest and remitted without treatment of any kind. Their capricious appearance and resolution suggested a mechanical lesion in the right side of the heart, possibly a pedunculated tumour such as a right atrial myxoma. Cor pulmonale and recurrent pulmonary embolization were also considered as alternatives. However, the patient died before a diagnosis was reached.

\section{Post-mortem}

The heart showed extensive patchy white endocardial fibrosis in the right ventricular outflow tract. On the right anterior cusp of the pulmonary valve there was a 1-cm papillary compound Lambl's excrescence, which resembled a sea-anemone when viewed under water (Fig. 1). The left side of the heart and the great vessels were normal. There was no evidence of pulmonary embolism or of recurrence of the treated breast carcinoma. The liver and kidneys were congested. There was considerable fibrosis of the left lung, and osteonecrosis of the left clavicle and third to fifth ribs, as a result of irradiation. No other abnormalities were found.

Microscopically the pulmonary valve lesion comprised multiple papillary avascular endothelialized fibrous processes, many containing rings of granular tissue which stained as elastic (Fig. 2). The features were typical of a papillary 'tumour' of heart valves (Pomerance and Davies, 1975).

\section{Discussion}

Papillary 'tumours' of the heart valves are now not 


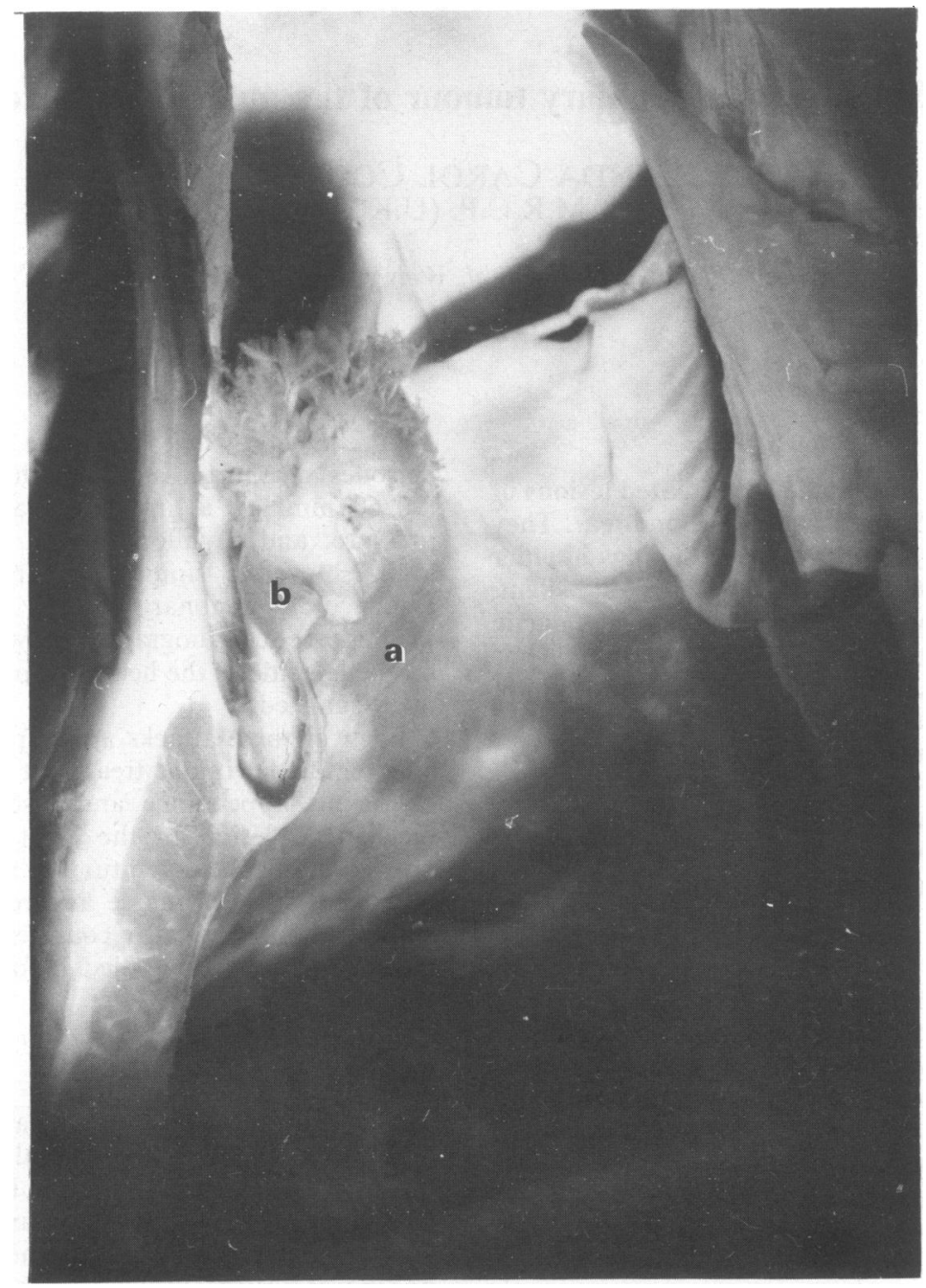

FIG. 1. Close-up of opened right ventricular outflow tract showing (a) patchy endocardial fibrosis, (b) right anterior cusp of pulmonary val:e (partly cut away) with papillary 'tumour' on the free edge. (HE, $\times 5)$.

uncommonly seen as an age-related finding and in patients with chronic valve disease. They are all small pedunculated lesions, covered by a single layer of endothelium continuous with that of the valve, or of the mural endocardium (Burn, Bishop, and Davies, 1969). Originally regarded as neoplastic, they are now believed to arise from the coalescence of Lambl's excrescences, which are small filiform tags up to $1 \mathrm{~cm}$ in length, the result of organization of partially attached deposits of fibrin on the surface of a valve (Magarey, 1949). Their occurence, and therefore that of papillary 'tumours', is thus related to thrombosis following endocardial damage such as rheumatic heart disease, and forms intermediate between Lambl's excrescences and papillaryo 'tumours' have been found (Pomerance, 1961).

Papillary 'tumours' arise most often on the left sides of the heart, frequently in association with hypertenfu sion, but have been observed on all valves (Pomerf ance, 1961; Nassar and Parker, 1971). They are్ usually asymptomatic incidental findings at post mortem (Dean and Falconer, 1914; Bohrod, 1929) or increasingly often, at cardiac surgery (Pomerance and Davies, 1975). Rarely when on the aortic valve they may cause angina by occluding the coronars ostia (Heath, Best, and Davis, 1961). Emboli into th 


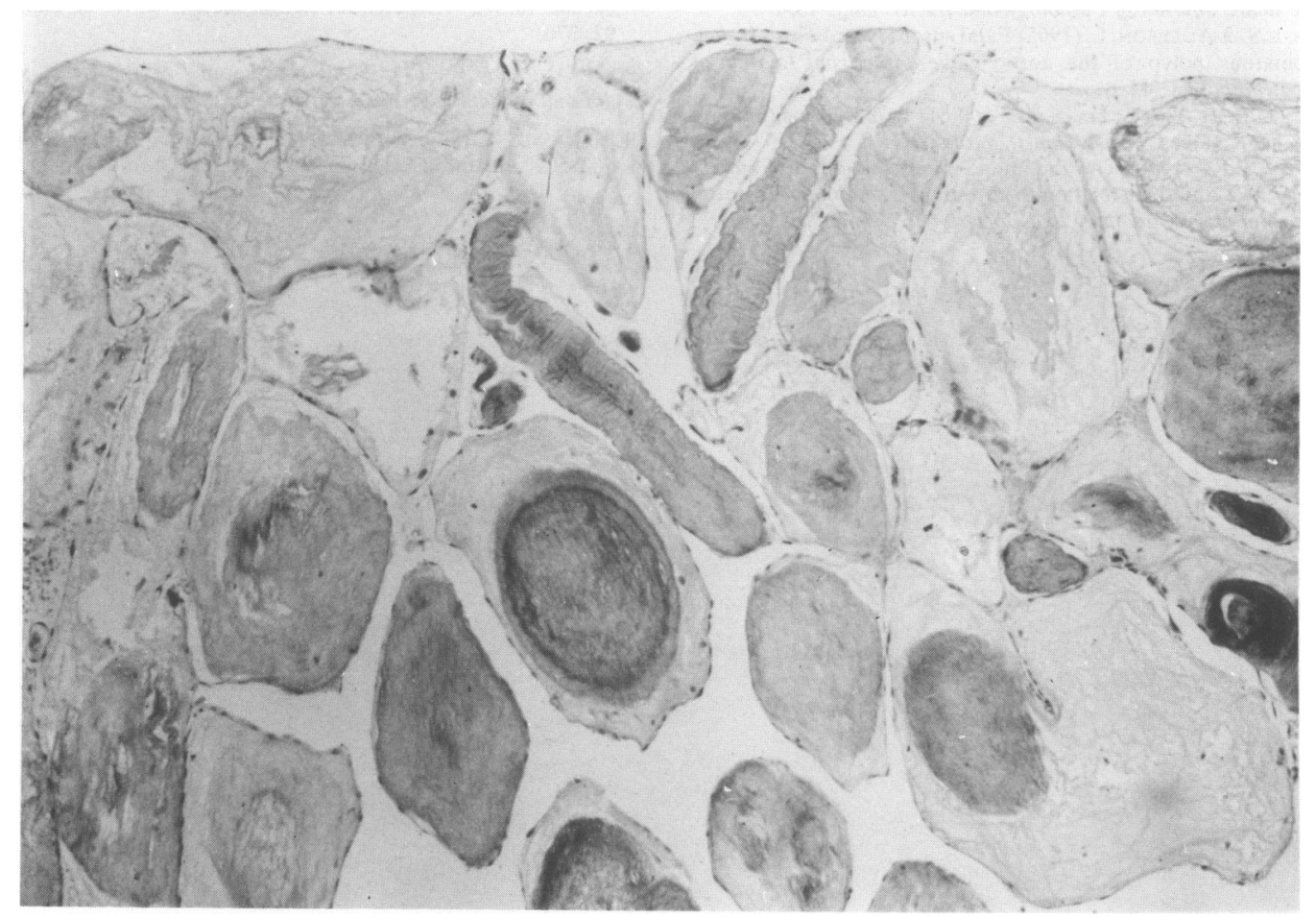

FIG. 2. Papillary 'tumour' showing multiple processes covered by a single layer of endothelium and concentric dark rings with the staining properties of elastic. $(\mathrm{HE}, \times 100)$.

coronary arteries (Harris and Adelson, 1965) and cerebral arteries (Nassar and Parker, 1971; Pomerance and Davies, 1975) have also been described.

Two reports concern lesions on the pulmonary valve (Catton, Guntheroth and Reichenbach, 1963) and tricuspid valve (Anderson, Fiddler and Lie, 1977) causing significant right ventricular outflow obstruction in infants with congenital heart disease. However these were probably not true papillary 'tumours', but rather examples of the myxoid dysplasia that often accompanies congenital heart disease. Indeed it is unlikely that such young children would have the opportunity to develop a lesion so dependent on wear-and-tear.

There has been no record to date of a pulmonary valve papillary 'tumour' causing symptoms in an adult. In the case reported here, the pulmonary artery pressure was not measured. Though pulmonary hypertension may have been expected as a sequel to extensive lung destruction, neither the electrocardiogram nor the post-mortem appearance of the great vessels suggested it. Intermittent right ventricular outflow obstruction is thus a more likely diagnosis than cor pulmonale. It is easy to visualize how this may result from episodic prolapse of a pedunculated lesion on the pulmonary valve. It is also highly probable that endocardial damage from radiotherapy was the main factor in the pathogenesis of this papillary 'tumour'.

\section{Acknowledgments}

I am indebted to Dr A. Pomerance for her help, Dr B. Jones for autopsy specimens, and Mr J. C. F. Townsend for allowing me to report on his patient.

\section{References}

ANDERSON, K.R., FIDdleR, G.I. \& LIE, J.T. (1977) Congenital papillary tumor of the tricuspid valve-an unusual cause of right ventricular outflow obstruction in a neonate with trisomy $E$. Proceedings of the Staff Meetings of the Mayo Clinic, 52, 665.

BoHROD, M.G. (1929) Multiple haemangiofibroma of the pulmonary valve. Archives of Pathology, 8, 68.

BURN, C.G., BishoP, M.B. \& DAVIES, J.N.P. (1969) A stalked papillary tumour of the mural endocardium. American Journal of Clinical Pathology, 51, 344.

CATTON R.W., GUNTHEROTH, W.G. \& REICHENBACH, D.D. (1963). A myxoma of the pulmonary valve causing severe stenosis in infancy. American Heart Journal, 66, 248. 
Dean, G. \& Falconer, A.W. (1914) Primary tumours of the valves of the heart. Journal of Pathology and Bacteriology, 18, 64.

HARR IS, L.S. \& ADELSON, L. (1965) Fatal coronary embolism from a myxomatous polyp of the aortic valve. American Journal of Clinical Pathology, 43, 61.

Heath, D., Best, P.V. \& Davis, B.T. (1961) Papilliferous tumours of the heart valves. British Heart Journal, 23, 20

MAGAREY, F.R. (1949) On the mode of formation of Lambl's excrescences and their relation to chronic thickening of the mitral valve. Journal of Pathology and Bacteriology, 61, 203.
NASSAR, S.G.A. \& PARKER, J.C. (1971) Incidental papillary endo- 르 cardial tumor-its potential significance. Archives of Pathology, $>$ 92, 370.

Pomerance, A. (1961) Papillary tumours of the heart valves. $\subseteq$ Journal of Pathology and Bacteriology, 81, 135.

Pomerance, A. \& Davies, M.J. (1975) Pathology of the Heart, pp. 500-502. Blackwell Scientific Publications, Oxford. 